\title{
Diffraction of Electromagnetic Waves on a Waveguide Joint
}

\author{
Mikhail Malykh ${ }^{1}$, Leonid Sevastianov ${ }^{1}$, Anastasiya Tyutyunnik ${ }^{1, \star}$, and Nikolai Nikolaev² \\ ${ }^{1}$ Department of Applied Probability and Informatics, Peoples' Friendship University of Russia \\ (RUDN University) \\ ${ }^{2}$ Department of Applied Physics, Peoples' Friendship University of Russia (RUDN University)
}

\begin{abstract}
In general, the investigation of the electromagnetic field in an inhomogeneous waveguide doesn't reduce to the study of two independent boundary value problems for the Helmholtz equation. We show how to rewrite the Helmholtz equations in the "Hamiltonian form" to express the connection between these two problems explicitly. The problem of finding monochromatic waves in an arbitrary waveguide is reduced to an infinite system of ordinary differential equations in a properly constructed Hilbert space. The calculations are performed in the computer algebra system Sage.
\end{abstract}

\section{Introduction}

The mathematical theory of the waveguide systems, based on the works of A.N. Tikhonov, A.A. Samarsky [1] and A.G. Sveshnikov [2], created in the middle of the last century. It has become an important chapter of the computational electrodynamics. The solution of waveguide diffraction problems is necessary for the design of integrated optical and fiber optic devices. The linear dimensions of these devices along different directions may differ by several orders of magnitude, for example, the radius of the Lüneburg thin-film waveguide lens exceeds its thickness by more than 104 times. Such a difference in size leads to insurmountable problems in the discretization of continuous models. The aim of our research is to overcome these difficulties. For example, the height of the Lüneburg waveguide lens is less than its diameter by $10^{4}$ times, which inevitably leads to specific computational peculiarities $[3,4]$. Therefore the development of modern program packages for the calculation of fields in the closed waveguides with extremal parameters remains an important challenge for the computational electrodynamics.

\section{Reduction of the system of Maxwell's equations to two scalar problems}

The investigation of the electromagnetic field in a regular waveguide filled with a homogeneous substance is reduced to the investigation of two independent boundary value problems for the Helmholtz equation [5]. In the case of a waveguide filled with an inhomogeneous substance, a relationship arises between the modes of these two problems, which in the numerical experiments can not always be fully taken into account. The first papers on the mathematical theory of the waveguide [6] had in mind the generalization of the Fourier method, therefore the question of reducing the system of Maxwell's

\footnotetext{
^e-mail: tyutyunnik_aa@pfur.ru
} 
equations to two scalar equations was in the shadow of the theorem on the completeness of the system of normal modes. Meanwhile, writing this pair is not at all difficult, if we go over to the "Hamiltonian form" of the Helmholtz equations.

Consider a waveguide of a constant simply connected section $S$ with ideally conducting walls. We will not make any starting assumptions with respect to the filling $\epsilon, \mu$ of this waveguide. The axis $O z$ is directed along the cylinder axis, the normal to $\partial S$ will be denoted as $\vec{n}$, the tangent vector perpendicular to $\vec{e}_{z}$ as $\vec{\tau}$. For a basis we take the system of Maxwell's equations, from which we exclude $E_{z}$ and $H_{z}$. For brevity's sake let us assume $\nabla=\left(\partial_{x}, \partial_{y}\right)^{T}$ and $\nabla^{\prime}=\left(-\partial_{y}, \partial_{x}\right)^{T}$. By excluding the longitudinal components from Maxwell's equations, we get

$$
\left\{\begin{array}{l}
\vec{e}_{z} \times \partial_{z} \vec{H}_{\perp}+\nabla \frac{1}{i k \mu}\left(\operatorname{curl} E_{\perp}\right)_{z} \times \vec{e}_{z}+i k \epsilon \vec{E}_{\perp}=0, \\
\vec{e}_{z} \times \partial_{z} \vec{E}_{\perp}-\nabla \frac{1}{i k \epsilon}\left(\operatorname{curl} H_{\perp}\right)_{z} \times \vec{e}_{z}-i k \mu \vec{H}_{\perp}=0 .
\end{array}\right.
$$

At the boundary, the conditions of ideal conductivity are satisfied to

$$
\vec{E}_{\perp} \times \vec{n}=0, \quad H_{\perp} \cdot \vec{n}=0, \quad E_{z}=0 .
$$

A two-dimensional analogue of the Helmholtz decomposition makes it possible, without loss of generality, to seek a solution of the system (1) in the form

$$
\vec{E}_{\perp}=\nabla u_{e}+\nabla^{\prime} v_{e}, \quad \vec{H}_{\perp}=\nabla v_{h}+\nabla^{\prime} u_{h} .
$$

The four scalar functions introduced here will be called potentials, and we'll always assume that they satisfy the boundary conditions $u_{e}=u_{h}=n \cdot \nabla v_{e}=n \cdot \nabla v_{h}=0$ at the boundary of the waveguide. Substituting these expressions into (1), we obtain

$$
\left\{\begin{array}{l}
\nabla^{\prime}\left(\partial_{z} v_{h}-\frac{1}{i k \mu} \Delta v_{e}\right)+i k \epsilon \nabla^{\prime} v_{e}-\nabla \partial_{z} u_{h}+i k \epsilon \nabla u_{e}=0, \\
\nabla^{\prime}\left(\partial_{z} u_{e}+\frac{1}{i k \epsilon} \Delta u_{h}\right)-i k \mu \nabla^{\prime} u_{h}-\nabla \partial_{z} v_{e}-i k \mu \nabla v_{h}=0 .
\end{array}\right.
$$

The first two of the three boundary conditions (2) are automatically satisfied. With the usual choice of basis, the third condition follows from the Dirichlet conditions imposed on the function $u_{h}$. If $\epsilon$ and $\mu$ are constants, then the system (3) can be rewritten as a pair of uncoupled Helmholtz equations with respect to $v_{e}, u_{h}$.

The presentation of a whole series of problems in the mathematical theory of waveguides can be carried out uniformly if we consider the field as a function of $z$ with values in a suitably chosen Hilbert space [7]. In other words, we will use the weak formulation of the problem of finding the potentials in the general case.

We will consider the solution

$$
w=\left(v_{h}, v_{e}, u_{e}, u_{h}\right)
$$

of the system (3) as a map of $z \in \mathbb{R}$ to a Hilbert space

$$
\mathfrak{H}=\left\{v_{h}, v_{e}, u_{e}, u_{h} \in W_{2}^{2}(S): u_{e}, u_{h}, n \cdot \nabla v_{e}, n \cdot \nabla v_{h}=0 \text { on } \partial S\right\} .
$$

In this space the equations of the system (3) can be written as follows

$$
\partial_{z} b(\tilde{w}, w)+i k a(\tilde{w}, w)+\frac{1}{i k} c(\tilde{w}, w)=0, \quad \forall \tilde{w} \in \mathfrak{H} .
$$


Here the forms $a, b, c$ are bounded in $\mathfrak{h}$, therefore, by the Riesz theorem, the above condition can be rewritten in terms of the operators generated by these bilinear forms. Thus any electromagnetic field in the waveguide satisfies the system of ordinary differential equations

$$
B \frac{d w}{d z}+i k A w+\frac{1}{i k} C w=0
$$

in the Hilbert space $\mathfrak{H}$. Since the embedding of the Sobolev spaces is compact, the operators $A$ and $B$ are compact, and $B$ is also self-adjoint.

\section{Normal modes of a waveguide}

Similar to the finite-dimensional case, any solution of the system (4) is a superposition of normal modes, that is, solutions that depend on $z$ as $e^{i k \beta z}$. To calculate these modes we obtain an eigenvalue problem with respect to the spectral parameter $\beta$ :

$$
\beta B w=A w-\frac{1}{k^{2}} C w .
$$

The discreteness of the spectrum and the completeness of the system of normal waves are obtained as a corollary of the theorem of M. V. Keldysh about the operator pencil [8].

The derivation of the numerical solution of this eigenvalue problem is divided into two steps:

- discretization, that is, truncation of the infinite matrices (the Galerkin method),

- solving the resulting algebraic eigenvalue problem.

In the standard approach to calculate the modes, we need to select some components of the vectors $\vec{E}$ and $\vec{H}$ and write an approximate problem for them using the finite element method or the finite difference method [9]. Our computing experiments have shown that the system (4) is more convenient for applying the Galerkin method because the truncation of operators $A, B$ and $C$ in this basis give us at once the algebraic eigenvalues problem in the form

$$
\beta \hat{B} w=\hat{A} w-\frac{1}{k^{2}} \hat{C} w
$$

The work proposed by us contains, in our opinion, an elegant and effective generalization of the incomplete Galerkin method [10]. In practice we calculate the matrix elements numerically with small errors but preserve all symmetry of the waveguide. As the 2nd step, the standard approach suggests using the Krylov Subspace Methods [11], but in modern computer algebra system Sage [12] we may work over the field of algebraic numbers without any numerical errors.

This approach to computing eigenmodes was tested on several waveguides [13]. Our computing experiments have shown that in such a way we can calculate all the running modes (that is, modes with real eigenvalues $\beta$ ) and the small locked modes with correct multiplicities. The last circumstance is unexpected and indicates new application for symbolical calculations in numerical analysis.

\section{The joint of two waveguides}

Let the filling $\epsilon, \mu$ of the waveguide have a jump at $z=0$, so this plane is the joint of two waveguides. Any solution of the differential equation (4) at $z<0$ and at $z>0$ can be represented as the superposition of normal modes. The problem about the diffraction of the wave

$$
\sum_{\beta>0} F_{n} w_{n}^{(1)} e^{i k \beta_{n}^{(1)} z}
$$


at the joint $z=0$ is reduced to the following: for the given waveguide, the functions $\epsilon_{1,2}(x, y)$ and $\mu_{1,2}(x, y)$ and the numbers $F_{1}, \ldots$, find numbers $R_{n}, T_{n}$ such that

$$
\sum_{\beta>0} F_{n} w_{n}^{(1)} e^{i k \beta_{n}^{(1)} z}+\sum_{\mathfrak{R} \beta<0} R_{n} w_{n}^{(1)} e^{i k \beta_{n}^{(1)} z}=\sum_{\mathfrak{R} \beta>0} T_{n} w_{n}^{(2)} e^{i k \beta_{n}^{(2)} z}
$$

at $z=0$. To solve this problem we can calculate the normal waves as above and solve a linear system with respect to $R$ and $T$ using the standard routine in computer algebra system (CAS).

\section{Conclusion}

We list the main results of our work.

- We can introduce four potentials, by means of which the system of Maxwell's equations in the waveguide reduces to an infinite system of linear ODEs and is convenient for numerical analysis by the Galerkin method.

- To compute the normal waves we can use CAS and save multiplicities and other qualitative properties of eigenvalues.

- The problem about diffraction on joint is reduced to a system of linear algebraic equations which can also be solved symbolically.

\section{Acknowledgments}

The calculations in this article are performed in the computer algebra system Sage [12]. The publication was prepared with the support of the "RUDN University Program 5-100". The work was partially supported by RFBF grants No 15-07-08795, No 16-07-00556.

\section{References}

[1] A.A. Samarskiy, Izbrannyye trudy (Moscow: MAX Press, 2003) 531 pp.

[2] I. E. Mogilevskii, A. G. Sveshnikov, Matematicheskiye zadachi teorii difraktsii (Moscow: Faculty of Physics MSU, 2010) 197 pp.

[3] A.A. Egorov, L.A. Sevastyanov, Kvantovaya Elektronika 39 (6), 566-574 (2009)

[4] A.A. Egorov et al., Kvantovaya Elektronika 40 (9), 830-836 (2010)

[5] W.C. Chew, Lectures on theory of microwave and optical waveguides (http://wcchew.ece.illinois. edu/chew/course/tgwAll20121211.pdf, 2012) 362 pp.

[6] A.A. Samarskiy, A.N. Tikhonov, Zhurnal tehnicheskoy fiziki 18 (7), 959-970 (1948)

[7] A.N. Bogolyubov, M.D. Malykh, Computations mathematics and mathematical physics 43 (4), 560-563 (2003)

[8] A.N. Bogolyubov, A.L. Delitsyn, A.G. Sveshnikov, Computational mathematics and mathematical physics 38 (11), 1815-1823 (1998)

[9] K.S. Chiang, Optical and Quantum Electronics 26, S113-S134 (1994)

[10] A.N. Bogolyubov, A.I. Erokhin, I.E. Mogilevskii, Computational Mathematics and Mathematical Physics 52 (6), 932-936 (2012)

[11] Y. Saad, Numerical methods for large eigenvalue problems (Philadelphia, PA : Society for Industrial and Applied Mathematics, 2011) 276 pp.

[12] SageMath, http://www.sagemath.org

[13] M. D. Malykh, L. A. Sevastianov, A. A. Tiutiunnik, N. E. Nikolaev, Journal of Electromagnetic Waves and Applications, 1-13 (2017) 